FinAL

Report on the DOE supported

\title{
R \& D PROGRAM FOR HADRON CALORIMETRY FOR THE SUPERCONDUCTING SUPER COLLIDER
}

\author{
DE-FG03-88ER40466 \\ Period 9/1/1988 - 6/30/1990 \\ Hans P. Paar \\ Physics Department 0319 \\ University of California, San Diego \\ La Jolla, CA 92093-0319
}

\section{DISCLAIMER}

\begin{abstract}
This report was prepared as an account of work sponsored by an agency of the United States Government. Neither the United States Government nor any agency thereof, nor any of their employees, makes any warranty, express or implied, or assumes any legal liability or responsibility for the accuracy, completeness, or usefulness of any information, apparatus, product, or process disclosed, or represents that its use would not infringe privately owned rights. Reference herein to any specific commercial product, process, or service by trade name, trademark, manufacturer, or otherwise does not necessarily constitute or imply its endorsement, recommendation, or favoring by the United States Government or any agency thereof. The views and opinions of authors expressed herein do not necessarily state or reflect those of the United States Government or any agency thereof.
\end{abstract}




\section{RESEARCH ACTIVITIES}

\subsection{OVERVIEW}

The University of California, San Diego (UCSD) group have completed the following projects during the period $9 / 1 / 1988-6 / 30 / 1990$ :

1. The analysis and publication ${ }^{[1,2,3,4,5]}$ of our SPAghetti CALorimeter (SPACAL) R \& D through the July 1989 beamtests.

2. The construction of a 2 ton scintillating fiber and lead prototype calorimeter with parallel geometry, consisting of 20 towers, each of hexagonal cross section ( $85 \mathrm{~mm}$ apex-to-apex) and $2 \mathrm{~m}\left(10 \lambda_{I}\right)$ long.

3. The construction (at UCSD) of a first tower with projective geometry.

4. Beamtests with the 2 ton prototype and the projective geometry prototype in two periods of one week each in. December 1989 and Spring 1990.

5. The construction of a 20 ton scintillating fiber and lead prototype calorimeter with parallel geometry of the same design as 2) above but with 155 towers. This prototype is large enough to fully contain hadronic showers.

6. Beamtests with the 20 ton prototype from. June 4-11, 1990.

7. The construction of two teststations for fiber measurements and for optical coupling and readout developments.

8. Conceptual design of a scintillating fiber and lead calorimeter in collaboration with Martin Marietta, Astronautics Division, Denver CO. This design has found its way into the EMPACT Expression of Interest ${ }^{[6]}$ that was submitted to the SSC laboratory.

9. Participation in the discussions of SPACAL based calorimetry for four potential SSC experiments: SDC, L*, EMPACT, and TEXAS. Three of these four collaborations have opted for the SPACAL technique as one of their options in their Expression of Interest. 
In progress are:

10. Construction of more prototype projective towers.

11. Studies of electron-hadron discrimination without longitudinal segmentation in the calorimeter, using the testbeam data obtained with the 2 ton and 20 ton prototypes. Both isolated electrons ${ }^{[7]}$ and electrons impacting near hadrons are studied.

12. Analysis of the data obtained in the recent beamtests in order to more precisely determine the characteristics and limitations of the SPACAL technique.

13. Conceptual mechanical design at UCSD of a scintillating fiber and lead calorimeter specifically for the SDC experiment. We build upon the results obtained in our collaboration with Martin Marietta; we use the same engineering tools.

With our work on the projects numbered $2,3,4,5,6,7,10,11$, and 12 above we are successfully fulfilling UCSD's obligations as described in the proposal and the Memorandum of Understanding.

\subsection{RESULTS}

Most of UCSD's prototype R \& D work is done within the SPACAL collaboration in which UCSD, CERN, and several other European Institutions participate. The 2 ton and 20 ton prototypes were assembled at CERN near the testbeams. UCSD has contributed 12 man-months of effort to this from a technician, two graduate students, and two Ph.D.s. Our trips to CERN were generally scheduled to coincide with one of the restbeam periods.

At UCSD we have worked on all aspects of the analysis of the beamtests of the prototypes but especially on electron-hadron discrimination for isolated and non-isolated electrons. We try to obtain sufficient hadron rejection without the traditional longitudinal segmentation of the calorimeter. This issue is a vital one 
for the success of the SPACAL technique. We have very exciting results indicating that one can indeed obtain the required hadron rejection under these conditions. In the case of isolated electrons, we make a lateral shower size cut and a cut on the pulseheight of a pre-shower detector placed immediately upstream of the calorimeter. We show in Fig. 1 the pion rejection factor and the the corresponding electron efficiency as a function of a cut on the pre-shower detector pulseheight. It is seen that pion rejection factors of up to several thousand can be obtained, a factor 10 better than is generally required for the SSC. As a second example we show in Fig. 2 our results for recognizing an electron near a hadron. We have used $D_{0}$ 's $H$-matrix method ${ }^{[8]}$ to define a $\chi^{2}$ for a given shower to be hadronlike in its spatial extent. The dark histcoram (use the left scale) shows the $\chi^{2}$ distribution for a thousand $80 \mathrm{GeV}$ pions. We compare this with the $\chi^{2}$ obtained for one thousand events, each of which was generated by overlapping one event from a $40 \mathrm{GeV}$ electron run with one event from a $40 \mathrm{GeV}$ pion run. The distance between the electron and pion impact points in the mixed events was only $43 \mathrm{~mm}$ ! The result is the open histogram (use right scale). It is seen that a $\chi^{2}$ cut at 500 recognizes $90 \%$ of the electron-pion overlap events as such while about 1:100 pion events are erroneously flagged as being a pion with a nearby electron.

As an example of other results obtained, we show in Fig. 3 the (in-)famous $e / \pi$ ratio as function of the cluster size (the shower energy is calculated by summing over a cluster of towers within a cylinder of radius $r$ ) for 20,40 , and $80 \mathrm{GeV}$. Fortunately for applications at the SSC, it is seen that one can sum over clusters with a radius as small as $30 \mathrm{~cm}$ and get an acceptable $e / \pi$ ratio. We show in Fig. 4 and 5 the $e / \pi$ ratio and the hadronic energy resolution respectively as a function of the ADC gate length. Again fortunately for applications at the $\mathrm{SSC}$, it is seen that one can use gate width as short as $50 \mathrm{~ns}$ and obtain adequate results.

These results show that the SPACAL technique is a promising one for the high rate and high particle density environment at the SSC. They also show that it is not necessary to use uranium as absorber in order to get compensation. 
Our data are unique in that they allow studies, in much more detail than ever before, of the properties of scintillator and lead hadron calorimeters. We mention response to jets (simulated by placing a thick target in the testbeam just upstream of the calorimeter), further studies of the compensation mechanism, dynamic range, noise, linearity, lineshape, and uniformity of response and resolution as function of the impact point and incident angle. More beamtests with the 20 ton detector are scheduled for Spring 1991.

We have spent much effort in the past year presenting the advantages and disadvantages of the SPACAL technique to four potential high $p_{T}$ SSC experiments. Questions were raised by our colleagues that we then addressed in our R \& D program. We mention electron-hadron separation, muon energy loss detection, and radiation damage. As a result three $\left(\mathrm{EMPACT}^{[\theta]}, \mathrm{SDC}^{[9]}\right.$, and TEXAS $^{[10]}$ )

have chosen the scintillating fiber and lead calorimeter as one of their options. This notwithstanding the fact that our calorimeter technique is a relatively new one (it was first proposed ${ }^{(11)}$ in April 1987). The R \& D has thus far been done with very limited resources compared to what other techniques like liquid argon or warm liquids have had at their disposal.

\section{INFRASTRUCTURE AT UCSD}

\subsection{LABORATORY}

In support of our prototype design, an undergraduate (Miss Tracy Taylor) runs electromagnetic shower simulations (EGS4) on the CRAY-YMP of the UCSD computer Center. The UCSD Supercomputer Center supports Tracy's salary and provides Supercomputer time at no charge to the D.O.E. contract.

Dr. Hans Paar and two undergraduates (Messrs. Noel Gonzales and Bob Schreiner) have worked since December 1, 1989 with a group of a half dozen engineers, analysts, and designers from Martin Marietta Astronautics Group in 
Denver on a conceptual mechanical design of a scintillating fiber calorimeter for EMPACT. Martin Marietta provided their manpower at no cost to the D.O.E. contract. Martin Marietta uses a commercially available Computer Aided Design system (the IDEA-S package ${ }^{[12]}$ that is also in use at Fermilab and SLAC). The UCSD Supercomputer Center has recently made this software package (licensing fee $\$ 10,000$ per year) available to us at no cost to the D.O.E. contract. We are currently exchanging design drawings by computer tape between Martin Marietta and UCSD. Dr. Mike Sivertz and Messrs. Noel Gonzalez and Bob Schreiner are currently working on a similar conceptual mechanical design for SDC.

Upon delivery of fiber from Kyowa (Japan), quality control tests are performed routinely, using test equipment developed especially for this purpose by our UCSD group using funds provided by D.O.E. in previous contract years. A graduate student (Mr. Beale Ong) and an undergraduate (Mr. Bob Schreiner) are in charge.

Calorimeter tower construction, using special tooling developed by our UCSD group, again with D.O.E. support, takes place with the help of our campus machine shop. One Ph.D. physicist (Dr. Mike Sivertz) and an undergraduate (Mr. Noel Gonzalez) are in charge.

Before leaving our group, Dr. Thomas has developed, jointly with our campus machine shop, a novel way to put grooves with the required precision in lead plates. He has given a presentation on this at the March 1990 meeting of the SSC Industrial Symposium in Miami, Florida.

Upon completion and prior to beamtests, the responses of calorimeter towers are tested by means of a remotely controlled U.V. lightsource. This equipment has also been developed by our UCSD group using funds provided by D.O.E. in previous contract years. A graduate student (Mr. Darin Acosta) and an undergraduate (Mr. James Piñon) are in charge.

$\mathrm{A}^{60} \mathrm{Co}$ ringsource for the calibration of towers is in the design stage with con- 
struction to start in September 1990. An undergraduate (Mr. Roger Gedminas) is in charge.

Analysis and design work takes place on VAX workstations that are situated in our laboratory and that are networked with UCSD's Physics Department VAX cluster for tape-, printer-, and system's services.

All members of the team participate in all aspects of our R \& D effort, the names given above indicate who are responsible for the proper execution of the various tasks.

\subsection{CURRENT PERSONNEL AT UCSD}

The following personnel are currently working on the project with the fraction of time indicated between parentheses:

1. Ph.D. physicists:

Dr. Hans P. Paar (75\%), Dr. Mike Sivertz (90\%),

2. Graduate students:

Mr. Darin Acosta (90\%), Mr. Beale Ong (90\%).

3. Undergraduate students:

Mr. Roger Gedminas (35\%), Mr. Noel Gonzalez (25\%), Mr. James Piñon (35\%), Mr. Bob Schreiner (35\%), Miss Tracy Taylor (35\%).

Miss Tracy Taylor is supported by the UCSD Supercompiter Center (NSF), Messrs. Noel Gonzalez and Mr. James Piñon by the UCSD Undergraduate Research Program. Messrs. Roger Gedminas and Bob Schreiner are supported by our D.O.E. contract.

Dr. Don Thomas has spent a sabbatical year with our group during the 1989 calendar year and since then has been partially supported by the DOE grant and continued to work with our group in 1990. 


\section{REFERENCES}

1) A Novel Way of Electron Identification in Calorimeters.

R. Desalvo et al. (SPACAL collaboration), Nuclear Instruments and Methods A279, 467 (1989).

2) Status of Scintillating Fiber Calorimetry.

H.P. Paar, Proceedings of the ECFA Study Week on Instrumentation Technology for High-Luminosity Hadron Colliders, p. 207, Barcelona, Spain, 14-21 September 1989, edited by E. Fernandez and G. Jarlskog.

3) Status of Scintillating Fiber Calorimetry.

M. Sivertz, Proceedings of the Division of Particles and Fields Conference, Houston TX, January 1990.

4) Results of Prototype Studies for a Spaghetti Calorimeter.

D. Acosta et al. (SPACAL collaboration), Nuclear Instruments and Methods A294, 193 (1990).

5) New Results in Scintillating Fiber Calorimetry.

M. Sivertz, Proceedings of the Tucson SSC Workshop, February 1990.

6) Electron Muons Partons with Aircore Toroids (EMPACT), R. Steiner et al. (with H.P. Paar from UCSD).

7) D. Acosta et al. (SPACAL collaboration), Electron-Pion Discrimination with a Scintillating Fiber Calorimeter, to be submitted the Nuclear Instruments and Methods.

8) R. Engelmann et al., Nuclear Instruments and Methods 216, 45 (1983).

9) Solenoidal Detector Collaboration (SDC), Expression of Interest, E.L. Berger et al. (with D. Acosta, B. Ong, and M. Sivertz from UCSD). 
10) TEXAS Collaboration, Expression of Interest, S. Dye et al.

11) The High Resolution Spaghetti Hadron Calorimeter.

P. Jenni, H.P. Paar, P. Sonderegger, R. Wigmans, NIKHEF report NIKHEF-H/87-7.

12) I-DEAS, Structural Dynamics Research Corporation, 2000 Eastman Drive, Milford, Ohio 45150. 


\section{FIGURE CAPTIONS}

1. Pion-electron separation at $80 \mathrm{GeV}$ as function of the cut on the preshower pulseheight, and the corresponding electron efficiency. Use the left scale for the pion fraction passing electron selection cuts (its inverse is the pion rejection factor) and the right scale for the electron efficiency.

2. $\chi^{2}$ distributions for the ' $80 \mathrm{GeV}$ pion hypothesis' applied to $80 \mathrm{GeV}$ pions (dark histogram, use the left scale) and applied to $40 \mathrm{GeV}$ electron and $40 \mathrm{GeV}$ pion overlayed events (open histogram, use the right scale). There are 1000 events in each histogram.

3. The $e / \pi$ ratio as function of the cluster size. The shower energy is calculated by summing over a cluster of towers within a circular cylinder of radius $r$.

4. The $e / \pi$ ratio as function of the $\mathrm{ADC}$ gate length.

5. The hadronic energy resolution $\sigma_{E} / E$ as a function of the $\mathrm{ADC}$ gate length. 


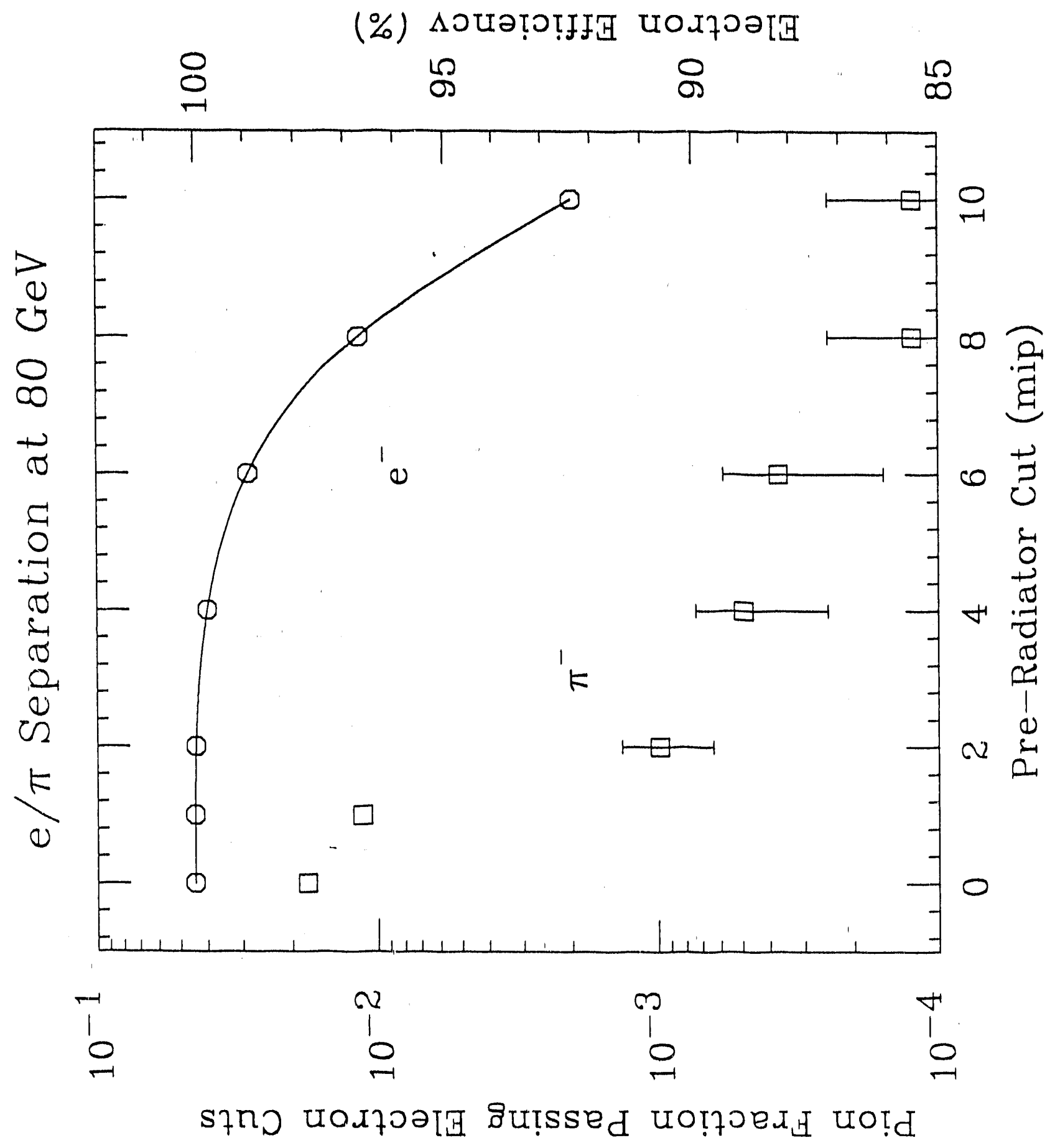

Figure 1 


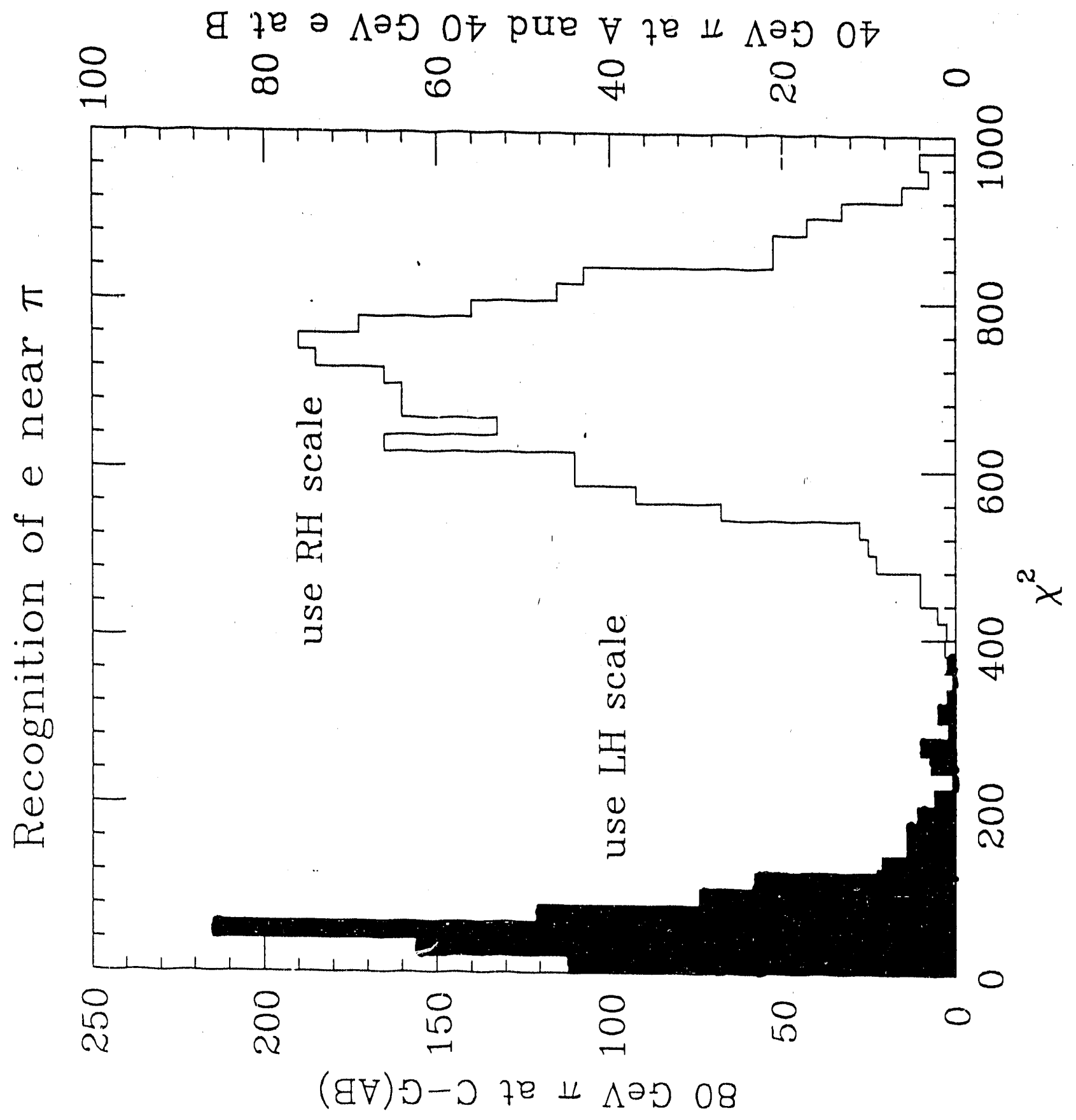

Figure 2 
SPACAL e/ $\pi$ as Function of Shower Containment

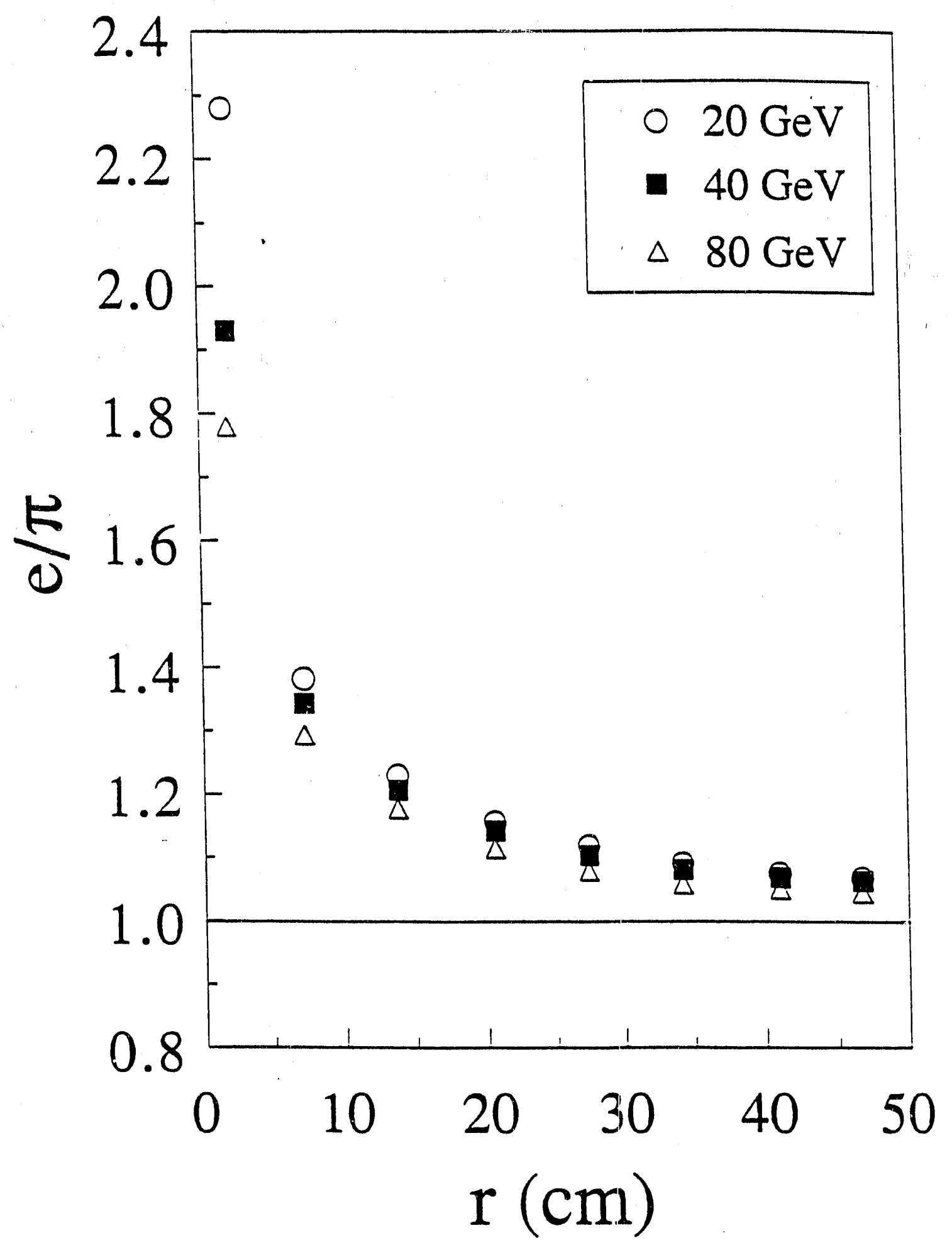

Figure 3 


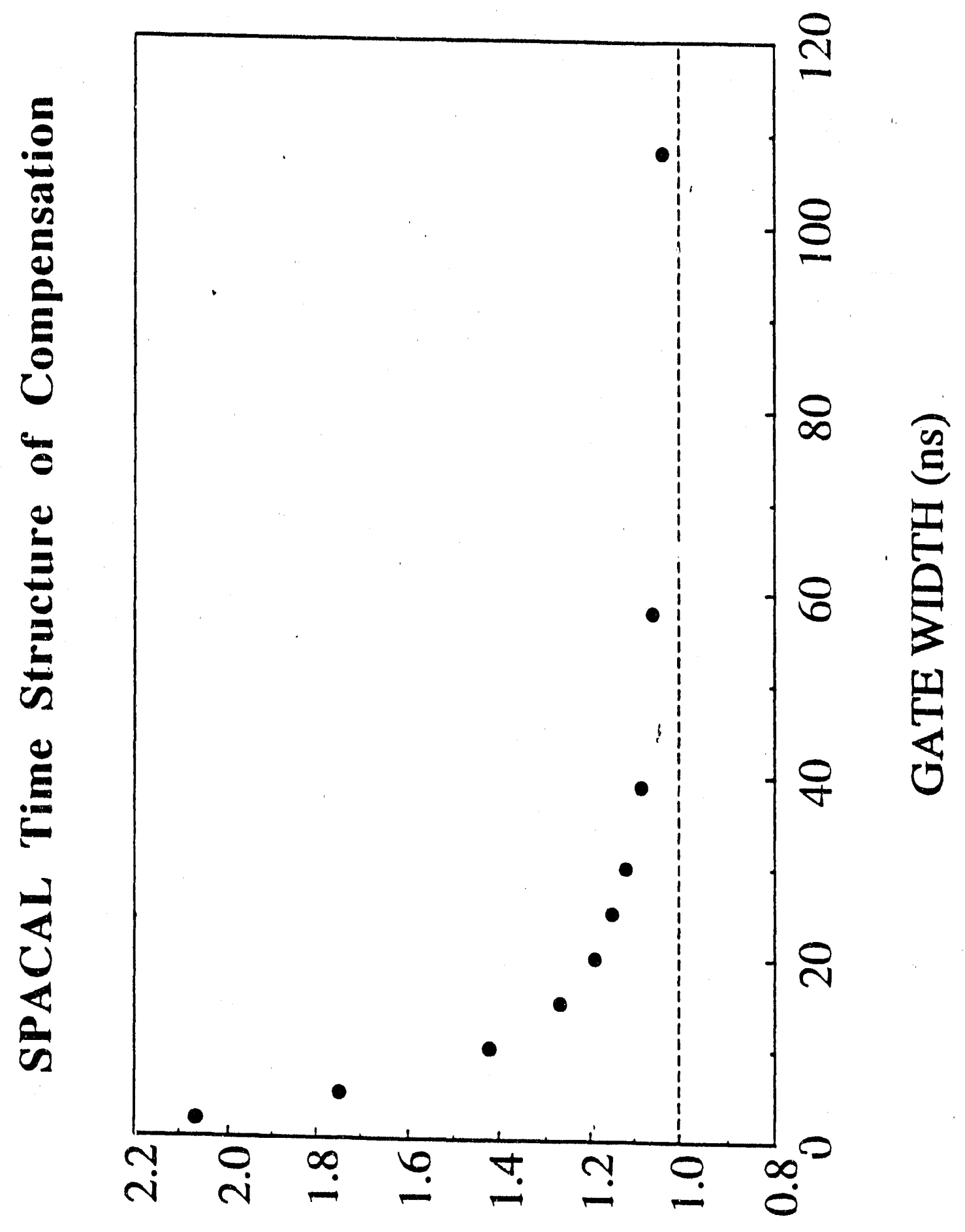

OILVY TVNOIS $1 /$

Figure 4 


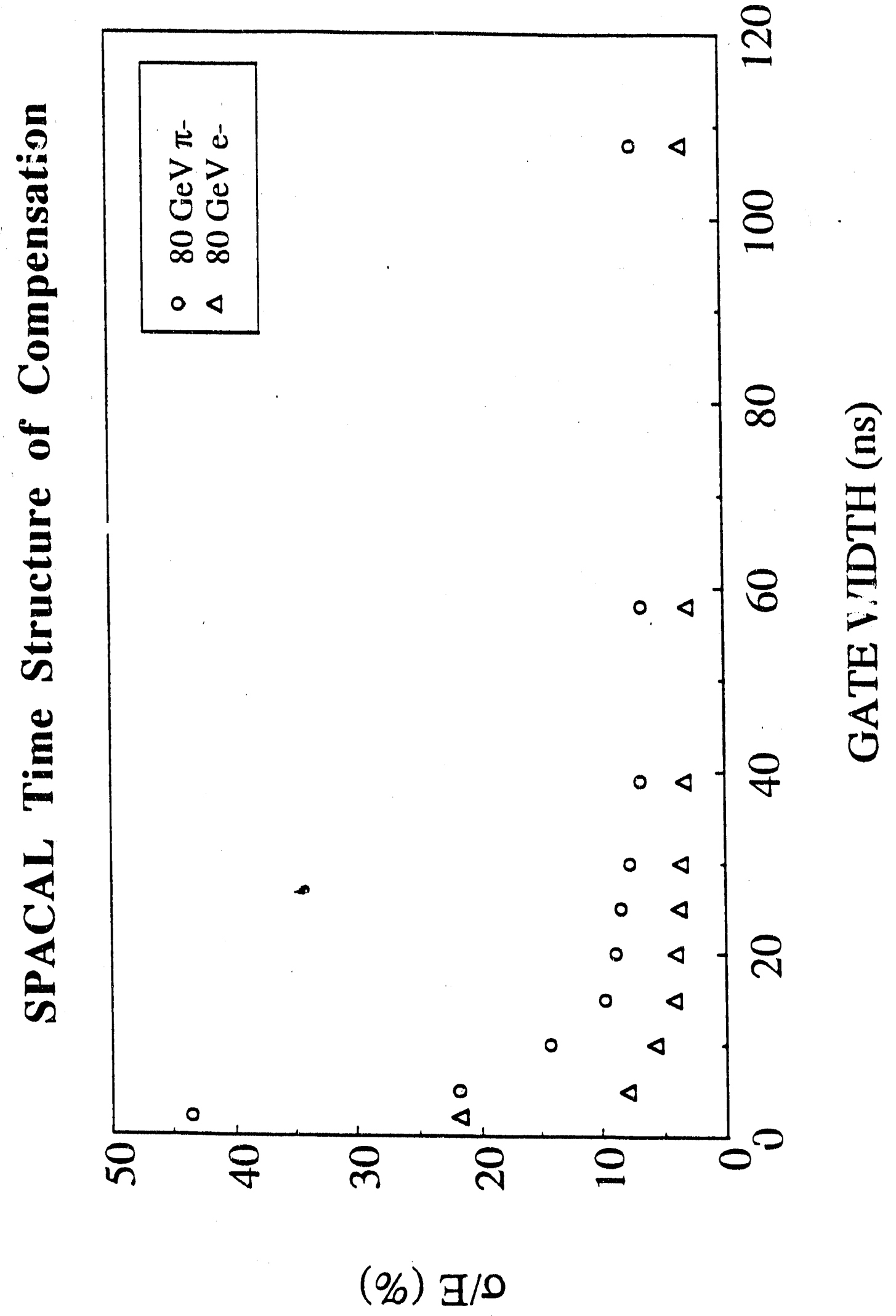

Figure 5 

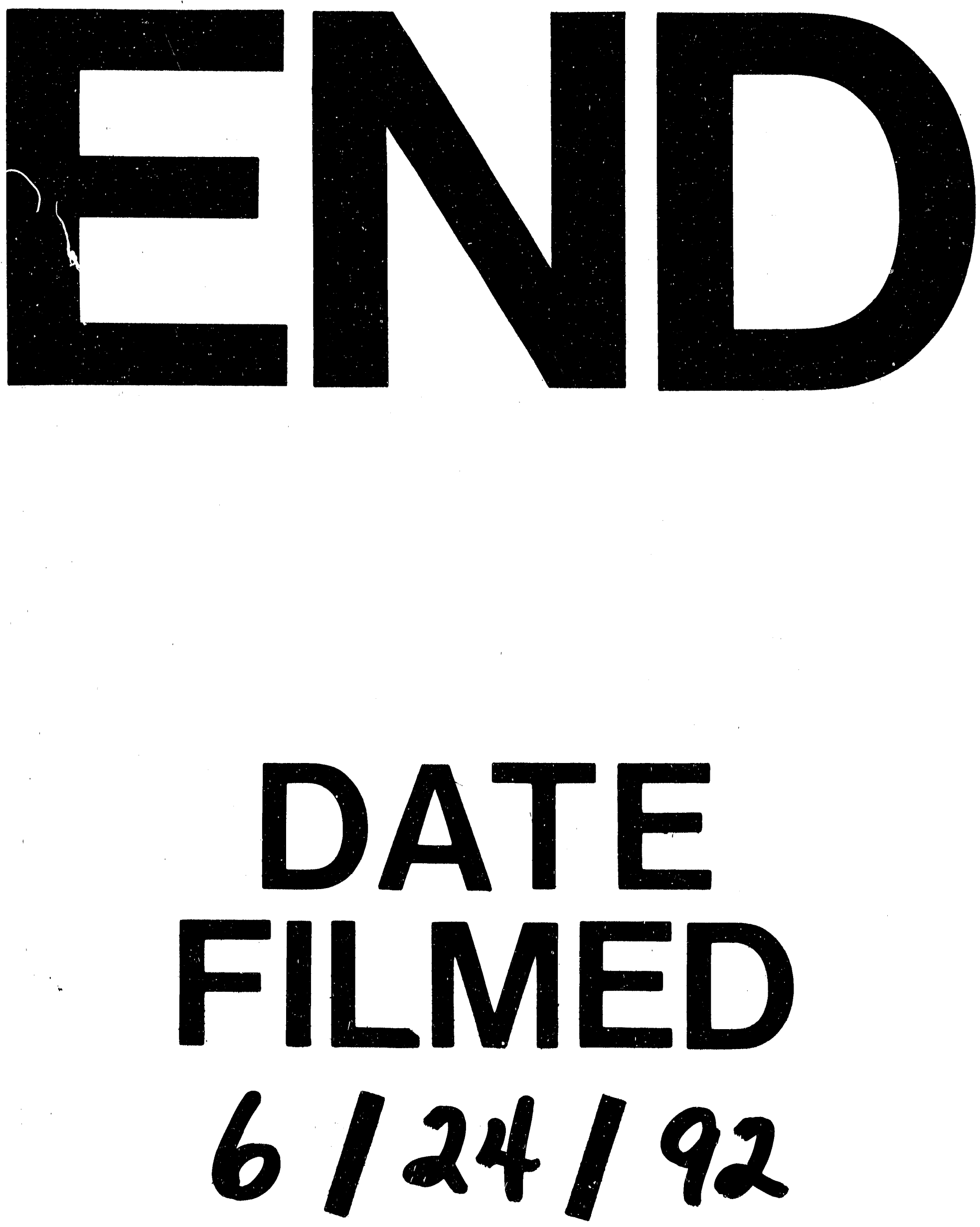
